OPEN ACCESS

Edited by:

Fabio Grizzi,

Humanitas Research Hospital, Italy

Reviewed by:

Mohamed Saad Zaghloul,

National Cancer Institute, Cairo

University, Egypt

Naokazu Ibuki,

Osaka Medical College, Japan

Hiroaki Matsumoto,

Yamaguchi University, Japan

*Correspondence:

Cora N. Sternberg

cnsternberg@corasternberg.com

Specialty section

This article was submitted to Genitourinary Oncology, a section of the journal Frontiers in Oncology

Received: 06 August 2018 Accepted: 02 October 2018 Published: 19 November 2018

Citation:

Del Bene G, Calabrò F, Giannarelli D,

Plimack ER, Harshman LC, Yu EY, Crabb SJ, Pal SK, Alva AS, Powles T,

De Giorgi U, Agarwal N, Bamias A,

Ladoire $S$, Necchi A, Vaishampayan UN, Niegisch G, Bellmunt J, Baniel J, Galsky MD and Sternberg CN (2018)

Neoadjuvant vs. Adjuvant

Chemotherapy in Muscle Invasive Bladder Cancer (MIBC): Analysis From the RISC Database.

Front. Oncol. 8:463

doi: $10.3389 /$ fonc. 2018.00463

\section{Neoadjuvant vs. Adjuvant Chemotherapy in Muscle Invasive Bladder Cancer (MIBC): Analysis From the RISC Database}

\author{
Gabriella Del Bene ${ }^{1}$, Fabio Calabrò ${ }^{1}$, Diana Giannarelli ${ }^{2}$, Elizabeth R. Plimack ${ }^{3}$, \\ Lauren C. Harshman ${ }^{4}$, Evan Y. Yu ${ }^{5}$, Simon J. Crabb ${ }^{6}$, Sumanta Kumar Pal ${ }^{7}$, Ajjai S. Alva ${ }^{8}$, \\ Thomas Powles ${ }^{9}$, Ugo De Giorgi ${ }^{10}$, Neeraj Agarwal ${ }^{11}$, Aristotelis Bamias ${ }^{12}$, \\ Sylvain Ladoire ${ }^{13}$, Andrea Necchi ${ }^{14}$, Ulka N. Vaishampayan ${ }^{15}$, Günter Niegisch ${ }^{16}$, \\ Joaquim Bellmunt ${ }^{4}$, Jack Baniel ${ }^{17}$, Matthew D. Galsky ${ }^{18}$ and \\ Cora N. Sternberg ${ }^{1 *}$ on behalf of the RISC Investigators
}

${ }^{1}$ San Camillo-Forlanini Hospital, Rome, Italy, ${ }^{2}$ IRCCS-Regina Elena Cancer Institute, Rome, Italy, ${ }^{3}$ Fox Chase Cancer Center, Philadelphia, PA, United States, ${ }^{4}$ Dana-Farber Cancer Institute, Harvard Medical School, Boston, MA, United States, ${ }^{5}$ Fred Hutchinson Cancer Research Center, University of Washington, Seattle, WA, United States, ${ }^{6}$ University of Southampton, Southampton, United Kingdom, ${ }^{7}$ City of Hope Comprehensive Cancer Center, Duarte, CA, United States, ${ }^{8}$ University of Michigan, Ann Arbor, MI, United States, ${ }^{9}$ St. Bartholomew's Hospital, London, United Kingdom, ${ }^{10}$ Istituto Scientifico Romagnolo per lo Studio e la Cura dei Tumori, IRCCS, Meldola, Italy, ${ }^{11}$ Huntsman Cancer Institute at the University of Utah, Salt Lake, UT, United States, ${ }^{12}$ National \& Kapodistrian University of Athens, Athens, Greece, ${ }^{13}$ Georges François Leclerc Cancer Center, Dijon, France, ${ }^{14}$ Istituto Nazionale Tumori of Milan, Milan, Italy, ${ }^{15}$ Karmanos Cancer Institute, Detroit, MI, United States, ${ }^{16}$ Department of Urology, Medical Faculty, Heinrich-Heine-University, Düsseldorf, Germany, ${ }^{17}$ Rabin Medical Center, Petah Tikva, Israel, ${ }^{18}$ The Tisch Cancer Institute, Icahn School of Medicine at Mount Sinai, New York, NY, United States

Background: $\mathrm{MIBC}$ is an aggressive disease, with 5-year survival rates ranging from 36 to $48 \%$ for p T3/p T4/p N+tumors. Perioperative treatment can improve overall survival, with more robust evidence in favor of neoadjuvant chemotherapy. Few randomized studies have compared neoadjuvant and adjuvant therapy in bladder cancer. Consequently, it has been difficult to establish the benefit of adjuvant chemotherapy (AC) in MIBC.

Methods: Data from patients with muscle invasive bladder cancer (>pT2) collected from 2005 to 2012 within the RISC data base (Retrospective International Study of Cancers of the Urothelial Tract) were evaluated. Overall survival (OS), cancer specific survival (CSS), and disease-free survival (DFS) between NC and AC generated using the Kaplan-Meier method were compared for MIBC by log-rank test. All patients in this analysis received either NC or AC.

Results: A total of 656 patients with MIBC (325 treated with AC and 331 with NC) were analyzed. The median DFS was 34.6 months (95\% Cl:25.3-43.9) for NC vs. 24.9 months (95\% Cl: 19.4-30.5) with $\mathrm{AC}$, with a reduction in the risk of disease progression of $21 \%$ in favor of NC (HR: 0.78, 95\% Cl: 0.63-0.96, $P=0.02)$. There were no significant 
differences in terms of CSS (HR: 1.06, 95\% Cl: 0.79-1.43, P: 0.70), and OS (HR: 1.08, 95\% Cl: 0.83-1.39, $P=0.57)$.

Conclusions: This study demonstrates superiority in DFS for NC compared to AC. The positive prognostic impact of complete pathological response to NC was confirmed.

Keywords: muscle invasive bladder cancer, neoadjuvant chemotherapy, adjuvant chemotherapy, RISC data base, locally advanced bladder cancer

\section{INTRODUCTION}

It is estimated that about 1.7 million new cases of cancer will be diagnosed in 2018 in the US with bladder cancer being the fourth most common cancer within males (1).

At the time of diagnosis, about $75-80 \%$ of bladder cancers are superficial, while the remaining $15-20 \%$ present as muscle invasive tumors. Approximately $50 \%$ of patients develop metastatic disease and in the past the median overall survival was dismal at 3-6 months without systemic treatment. The addition of cisplatin based therapy improved survival to between 12 and 15 months (2).

Despite improvement in surgical techniques, the rate of local and remote relapse remains high. The 5-year overall survival rates after radical cystectomy range from 36 to $48 \%$ for pT3-T4 and/or $\mathrm{pN} 0 / \mathrm{pN}+$ disease, most likely due to the presence of micro metastasis at the time of diagnosis (3).

Perioperative treatments either before or after surgery, can reduce the risk of both local and distant recurrence and increase overall survival.

Several studies and meta-analyses have been conducted, with more consistent results in favor of NC therapy, which is recommended for the treatment of MIBC (level 1 evidence). The lack of robust evidence for AC derives mainly from the difficulties in accrual and methodological problems of the trials that have been conducted in this setting. However, AC represents an important option for patients with MIBC (pT3-T4 and/orpN0 /pN+ disease) who have not received NC.

Few studies, mostly retrospective, have compared the two treatment strategies of $\mathrm{NC}$ and $\mathrm{AC}$, so data on the optimal sequence remains controversial. The aim of this analysis is to compare the efficacy of NC and AC treatment in MIBC, based on data from the RISC database.

\section{MATERIALS AND METHODS}

The Retrospective International Study of Cancers of the Urothelial Tract (RISC) is a population-based, retrospective study with the primary objective to describe the management, patterns of care and outcome of patients with urothelial cancer (clinical T-classification cT2 disease or greater). RISC consists of patient series from 28 international centers between 2005 and 2012 (some centers included patient data over this entire period, whereas others included data over a more limited number of years). Data concerning baseline characteristics, laboratory and pathology information, and treatment outcomes were collected using a password-protected, secure, web-based, electronic data capture tool. Investigators were trained using a web-based tutorial and supported by a comprehensive study training manual and data dictionary. The coordinating center, Icahn School of Medicine, Mount Sinai Hospital, New York, NY, curates the data with queries completed by each participating site. The study was approved by the ethics committees at each participating institution.

Patient inclusion criteria for the current analysis included a diagnosis of muscle invasive bladder cancer of any histology treated with $\mathrm{NC}$ ( $\geq \mathrm{cT} 2$, cN0, M0) followed by surgery (radical cystectomy), or the contrary, with cystectomy first, followed by $\mathrm{AC}(\geq \mathrm{pT} 2$, any $\mathrm{pN}, \mathrm{M} 0)$. Patients treated with adjuvant radiotherapy or a combination of radiation and chemotherapy were excluded, as well as those treated with both NC and AC.

The primary objectives were the comparison between NC and $\mathrm{AC}$, in terms of disease free survival (DFS), overall survival (OS), and cancer specific survival (CSS), which were calculated from the time of diagnosis of muscle invasive disease until disease progression, death from any cause or specifically from cancer, respectively. DFS, OS, and CSS were calculated with 95\% confidence intervals, with analysis of survival generated using the Kaplan-Meier method. The comparison between survival curves for every endpoint, was done with the log rank test (4). Hazard ratios and their 95\% CI were derived from the proportional hazard model and were estimated for type of chemotherapy (NC vs. AC), histology (urothelial vs. non-urothelial), pT and pN status, Charlson index, age, gender, and smoking habits. A propensity matched analysis was also performed, matching samples according to $\mathrm{pT}$ and $\mathrm{pN}$ status.

There is an issue about what to consider as time 0 for this kind of analysis. If defined by the initiation of chemotherapy, the NC patients would necessarily have had to live longer than AC patients. That is, NC patients by definition had to live about 3-4 months longer because their time 0 was the start of $\mathrm{NC}$, and by definition they had to survive to have cystectomy. On the other hand, time 0 for the AC patients would start months later at the start of chemotherapy.

At the same time, if it is considered the date of cystectomy as time 0 , it still may lead to an unfair advantage for the adjuvant patients: because the receipt of AC occurs after cystectomy and patients in the AC group are defined by the receipt of AC. This group by definition still has to live long enough to receive $\mathrm{AC}$ and longer than NC group. So, this can deprive the NC group of 3-4 months of survival and does not allow those who did not undergo radical cystectomy to be considered in calculations of survival rates. For this reason, we felt that the soundest methodology for 
this type of analysis was to use time of diagnosis of bladder cancer as time 0 for both NC and AC.

\section{RESULTS}

From 2005 to 2012 a total of 3,024 patients with urothelial cancer were included in the RISC database. Overall, 1,892 of these patients underwent surgery for MIBC and UTUC. Seventy-four patients were excluded from the original sample due to lack of data, 139 due to incomplete data concerning perioperative therapy, 39 patients with lymph node metastatic disease $(\mathrm{cN}+)$ were excluded from the NC group, 875 were not considered because they had not received any perioperative treatment, while 32 were left out of the analysis because they underwent both treatments (NC and $\mathrm{AC}$ ).

Therefore, the final sample for the analysis consists of 656 patients with MIBC, treated with surgery and perioperative chemotherapy. Among patients with MIBC, 325 were treated with $\mathrm{AC}$ and 331 with NC (Figure 1).

Patient characteristics are detailed in Table 1. The majority of patients $(>70 \%)$ were male. Other patient characteristics, in particular with regard to age ( $<65$ years or $\geq 65$ years) and Charlson index, were equally distributed among the various groups.

The significant difference relative to the percentage of patients with clinically positive lymph nodes among the two groups, derives mainly from the need to exclude those patients from the NC group, as they had clear metastatic disease at the time of diagnosis and they should have been treated with more cycles of chemotherapy. Moreover, the differences between the $\mathrm{pT}$ and $\mathrm{pN}$ in the two groups may be due to down-staging as a result of neoadjuvant chemotherapy.

More than $70 \%$ of patients were smokers or former smokers, and most of them had pure urothelial histology $(>70 \%)$ in each group. Mixed histology (urothelial with other features: squamous, neuroendocrine, sarcomatoid, micropapillary, etc.) was observed in $21.9 \%$ of patients. Non-urothelial histologies were found in $23.2 \%$ of patients.

The majority of patients, both in the NC and AC setting, were treated with combination cisplatin based chemotherapy. In particular, in the MIBC NC group, $74.9 \%$ of patients were treated with cisplatin based chemotherapy, while $22.1 \%$ received treatment without cisplatin. In the MIBC AC group, $71.1 \%$ received cisplatin based therapy vs. $23.1 \%$ non-cisplatin based. The different chemotherapy regimens used are shown in the Appendix in Supplementary Material.

The median DFS was 34.6 months (95\% CI: 25.3-43.9) for NC vs. 24.9 months (95\% CI: 19.4-30.5) with AC, with a reduction in the risk of disease progression of $21 \%$ in favor of NC (HR: 0.78 , 95\% CI: 0.63-0.96, $P=0.02$; Figure 2).

The median cancer specific survival (CSS) was 115.2 months (95\% CI: 30.3-200.1) for NC vs. 92.8 months (95\% CI: 73.3-112.3) for AC. This difference was not statistically significant (HR: 1.06, 95\% CI: 0.79-1.43, $P=0.70$; Figure 3).

The median overall survival (OS) was of 51.7 months (95\% CI:37.8-65.6) for the NC group and 66.8 months (95\% CI:
51.1-82.5) for AC. The difference was not statistically significant (HR: 1.08 ; $95 \%$ CI $0.83-1.39, P=0.57$; Figure 4).

There was a significant difference in DFS and OS between patients with complete pathologic response (pT0) after NC compared to those with residual disease $(\geq \mathrm{pT} 1$; DFS at 5 years pT0 vs. $\geq$ pT1: 62 vs. $21 \%$, respectively, $P<0.0001$; OS at 5 years pT0 vs. $\geq$ pT1: 72 vs. $31 \%$, respectively, $P<0.0001$; Appendix, Supplementary Survival Curves).

There was longer DFS and OS for NC in MIBC for patients without lymph node metastasis, compared to those with lymph node involvement (DFS 5 years pN0 vs. $\mathrm{pN}+: 47$ vs. $9 \%$, respectively, $P<0.0001$; OS 5 years pN0 vs. pN+: 51 vs. $25 \%$, respectively, $P<0.0001$; Appendix, Supplementary Survival Curves).

In the AC group, there was a significant difference in DFS $(P=0.001)$ and $\mathrm{OS}(P<0.0001)$ at 5 years in relation to $\mathrm{pT}$ status, with better results for lower stages than more advanced stages (pT2 vs. pT3 vs. pT4), while there was no significant difference for DFS $(P=0.11)$ or OS $(P=0.29)$ in relation to pathological lymph node status ( $\mathrm{pN} 0$ vs. $\mathrm{pN}+$ ).

In the AC group, there was also better OS at 5 years for urothelial histology than for non-urothelial tumors, (56 vs. $43 \%$, $P=0.001)$, significant differences were observed also in DFS at 5 years (31 vs. $19 \%, P=0.002$ ).

A multivariate analysis of survival times (DFS, CSS, OS) was performed. The variables considered in the Cox regression model were sex, age, T, N, smoking habits, Charlson index, histology, and NC vs. AC. The hazard ratio of NC vs. AC revealed a positive effect on DFS for NC with respect to AC only in the univariate analysis, not in the multivariate analysis, and a negative effect for CSS and OS. A complete pathological response and the absence of lymph node metastasis had a significant positive impact on DFS, OS, and CSS in both multivariate and univariate analysis (Supplementary Table 1). In the multivariate analysis, the HRs (adjusted for $\mathrm{pN}$ and $\mathrm{pT}$ ) were in favor of $\mathrm{AC}$ for all the three survival endpoints.

When considering a propensity score based on the two prognostic factors ( $\mathrm{pT}$ and $\mathrm{pN}$ ) differences between $\mathrm{NC}$ and $\mathrm{AC}$ in DFS disappeared $(P=0.38)$; also differences in terms of CSS $(P=0.40)$ and $\mathrm{OS}(P=0.26)$ resulted not significant.

\section{DISCUSSION}

Muscle invasive bladder cancer is a highly aggressive disease, with a high rate of early metastatic dissemination, and a low 5-year overall survival rate. Perioperative cisplatin-based chemotherapy in addition to surgery to improve outcomes in high risk MIBC has shown increased disease control with improved survival, probably due to eradication of micrometastatic disease. Currently, the evidence favors NC, which has shown an advantage in terms of OS for cisplatin based combinations in at least two randomized trials and multiple meta-analyses.

In particular, the MRC BA06/EORTC 30894 trial, demonstrated a 10 -year benefit of $6 \%$ for NC, and the SWOG study by Grossmann et al, confirmed a trend toward better OS in favor of NC with M-VAC $(5,6)$. Moreover, in 2005, a 
3024 patients in RISC database

1892 patients underwent surgery

\section{I}

74 excluded due to lack of data

139 excluded due to incomplete

data on perioperative treatment

39 patients with $\mathrm{cN}+$ excluded

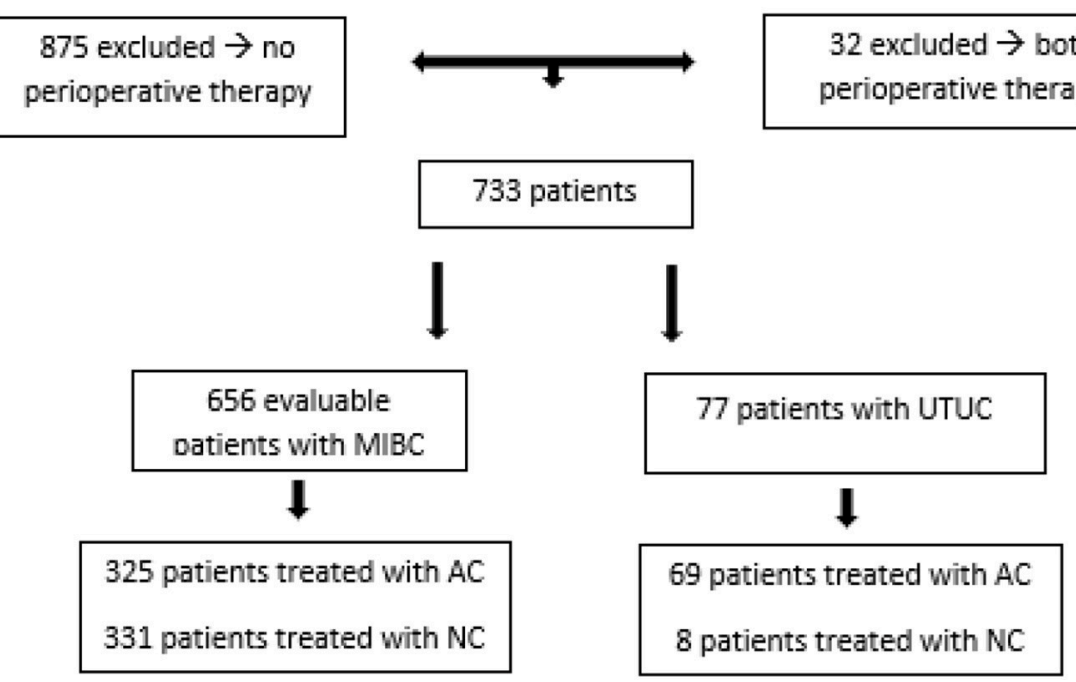

FIGURE 1 | Flow chart: patients captured from the RISC database. UTUC, Upper tract urothelial carcinoma. This group was not included in this analysis.

meta-analysis of 11 trials of NC encompassing 3,005 patients showed a reduction in the risk of death of $14 \%(\mathrm{HR}=0.86,95 \%$ CI $0.77-0.95, P: 0.003)$, with a survival advantage at 5 years of $5 \%$ (from 45 to $50 \%$ ) (7).

The role of $\mathrm{AC}$ is more controversial given data derived mainly from studies with inconsistent results due to methodological problems and inadequate patient numbers due to premature closure and poor recruitment. No single study, taken individually, has demonstrated a statistically significant benefit in survival in favor of AC with the exception of the Spanish study (Spanish Oncology Genitourinary GroupSOGUG). It showed a potential benefit in OS for AC with cisplatin, paclitaxel, gemcitabine (median OS 26 months; 5-year OS, $31 \%$; $P<0.0009)$, DFS $(P:<0.0001)$, TTP $(P:<0.0001)$, and CSS $(P:<0.0002)$. This study was closed prematurely due to poor recruitment, and the results were presented at an ASCO meeting but have never been fully published (8).

The EORTC performed the largest AC study to date (trial 30994). This study was also hampered by difficulties in enrolment. The study evaluated immediate AC with four cycles of chemotherapy vs. observation and six cycles of chemotherapy at the time of recurrence. GC, M-VAC or HD-VAC were allowed. This study did not show a benefit in OS (adjusted HR 0.78, 95\% CI.0.56-1.08; $P=0.13$ ), but did reveal a highly significant improvement in PFS, with 5 year PFS of 47.6 vs. $31.8 \%$ for those given immediate adjuvant chemotherapy (HR: 0.54, 95\% CI: $0.40-0.73, P<0.0001)$. There was, however, a benefit in OS for the subgroup of patients with negative lymph nodes (pN0) (9) and a non-significant $22.2 \%$ reduction in the risk of death with immediate adjuvant chemotherapy in the ITT population.

Meta-analyses have been conducted to clarify the role of AC. The analysis published in 2014 without the EORTC study data, showed a reduction in the risk of death with AC of $23 \%$ (HR 0.77, 95\% CI 0.59-0.99; P: 0.049) (10). A further update of this metaanalysis, conducted with the EORTC 30994 study, demonstrated a survival benefit with immediate adjuvant treatment (HR 0.77, 95\% CI 0.65-0.91; $P=0.002)(10,11)$.

Therefore, at present cisplatin-based combination $\mathrm{AC}$ is a valuable option for patients with bladder cancer pT3-pT4, $\mathrm{pN} 0 / \mathrm{pN}+, \mathrm{M} 0$ who have not received preoperative treatment. 
TABLE 1 | Patient characteristics.

\begin{tabular}{|c|c|c|c|}
\hline $\mathbf{N}^{\circ}$ of patients (\%) & NC MIBC (331) & AC MIBC (325) & $P$-value \\
\hline \multicolumn{4}{|l|}{ GENDER } \\
\hline Male & 257 (77.6) & $271(83.4)$ & 0.06 \\
\hline Female & 74 (22.4) & $54(16.6)$ & \\
\hline \multicolumn{4}{|l|}{ AGE } \\
\hline$<65$ years & $179(54.1)$ & $184(56.6)$ & 0.23 \\
\hline$\geq 65$ years & $145(43.8)$ & $139(42.8)$ & \\
\hline missing & $7(2.1)$ & $2(0.6)$ & \\
\hline \multicolumn{4}{|l|}{ CHARLSON } \\
\hline 0 & $154(46.5)$ & $133(41.0)$ & 0.34 \\
\hline $1-2$ & $90(27.2)$ & $107(32.9)$ & \\
\hline$\geq 3$ & $61(18.4)$ & $56(17.2)$ & \\
\hline Missing & $26(7.9)$ & $29(8.9)$ & \\
\hline \multicolumn{4}{|c|}{ SMOKING HISTORY } \\
\hline Current & $76(30.0)$ & $83(25.5)$ & 0.29 \\
\hline Former & $140(42.3)$ & $131(40.3)$ & \\
\hline Never & 89 (26.9) & $72(22.2)$ & \\
\hline Missing & $29(8.8)$ & 39 (12.0) & \\
\hline \multicolumn{4}{|l|}{ pT } \\
\hline 0 & $91(27.5)$ & $3(0.9)$ & $<! 0.0001$ \\
\hline 1 & $16(4.8)$ & $6(1.8)$ & \\
\hline 2 & $56(16.9)$ & 55 (16.9) & \\
\hline 3 & 87 (26.3) & $165(50.8)$ & \\
\hline 4 & 35 (10.6) & $77(23.7)$ & \\
\hline Ta & $6(1.8)$ & $1(0.3)$ & \\
\hline Tis & $21(6.3)$ & $1(0.3)$ & \\
\hline Missing & $19(5.7)$ & $17(5.3)$ & \\
\hline \multicolumn{4}{|l|}{$\mathrm{pN}$} \\
\hline 0 & $234(70.7)$ & $74(22.8)$ & $<! 0.0001$ \\
\hline+ & 68 (20.5) & $214(65.8)$ & \\
\hline$x$ & $9(2.7)$ & $14(4.3)$ & \\
\hline Missing & $20(6.1)$ & $23(7.1)$ & \\
\hline \multicolumn{4}{|l|}{ cT } \\
\hline 0 & 0 & $1(0.3)$ & 0.01 \\
\hline 1 & $6(1.8)$ & $15(4.6)$ & \\
\hline 2 & $183(55.3)$ & 169 (52.0) & \\
\hline 3 & $83(25.1)$ & $75(23.1)$ & \\
\hline 4 & $29(8.8)$ & $18(5.6)$ & \\
\hline $\mathrm{Ta}$ & 0 & $2(0.6)$ & \\
\hline Tis & 0 & $1(0.3)$ & \\
\hline Missing & $30(9.1)$ & $44(13.5)$ & \\
\hline \multicolumn{4}{|l|}{$\mathrm{cN}$} \\
\hline 0 & $219(66.1)$ & $135(41.6)$ & $<! 0.0001$ \\
\hline+ & 0 & $69(21.2)$ & \\
\hline$x$ & 79 (23.9) & $69(21.2)$ & \\
\hline Missing & $33(10.0)$ & $52(16.0)$ & \\
\hline \multicolumn{4}{|l|}{ HISTOLOGY } \\
\hline TCC & 237 (71.6) & 248 (76.3) & 0.10 \\
\hline TCC+other & 44(13.3) & $28(8.6)$ & \\
\hline Other & 35 (10.6) & $41(12.6)$ & \\
\hline Missing & $15(4.5)$ & $8(2.5)$ & \\
\hline
\end{tabular}

(Continued)
TABLE 1 | Continued

\begin{tabular}{lccc}
\hline N $^{\circ}$ of patients (\%) & NC MIBC (331) & AC MIBC (325) & $P$-value \\
\hline CT REGIMEN & & & \\
Cisplatin based & $248(74.9)$ & $231(71.1)$ & 0.18 \\
No CDDP based & $73(22.1)$ & $75(23.1)$ & \\
Missing & $10(3.0)$ & $19(5.8)$ &
\end{tabular}

TCC, Transitional cell carcinoma; CT, chemotherapy; CDDP, cisplatin. Other (nonurothelial histologies: adenocarcinoma, squamous cell carcinoma, sarcomatoid carcinoma, small cell carcinoma, pure micropapillary, urachal adenocarcinoma).

Currently, it is not possible to establish with absolute certainty what is the best sequence of perioperative treatments. The most persuasive evidence has been in favor of NC.

Until now, only one study compared NC with AC in a prospective manner. This study was conducted in 2001 at the MD Anderson Cancer Center. In this trial, 140 patients were randomized to receive preoperative treatment with two cycles of M-VAC followed by surgery and three additional M-VAC cycles, or immediate surgery followed by five AC cycles. At a median follow-up of 6.8 years, no statistically significant differences were observed in OS and DSS between the two treatment groups (12).

In a retrospective study at Columbia University, OS and DSS were analyzed in 146 patients who received perioperative therapy between 1988 and 2009 (73 neoadjuvant and 73 adjuvant) (11).

In this report, no statistically significant difference between the two treatments was observed. Another retrospective study in 42 patients, compared the combination of cisplatin and gemcitabine in the $\mathrm{NC}$ and $\mathrm{AC}$ setting without demonstrating any difference in recurrence free survival $(P: 0.124)(13)$. All of these results seem to suggest that the sequence of treatments surrounding cystectomy is less critical than the perioperative therapy itself.

The results of a retrospective study from the National Cancer Database were presented at the ASCO meeting in 2016. This study, based on a series of more than 1,600 patients treated with $\mathrm{NC}$ and 800 with $\mathrm{AC}$, compared $\mathrm{NC}$ to $\mathrm{AC}$ and to surgery alone, in terms of OS. Multivariate analysis showed higher OS ( $P$ : 0.008 ) for the patients treated with NC (14). These results are not conclusive given the retrospective nature of the work, but may suggest further caution in interpretation of the results of metaanalysis and large retrospective studies in favor of the role of $\mathrm{AC}$ $(10,15)$.

In our retrospective study, the comparison between $\mathrm{NC}$ and $\mathrm{AC}$ was done in a broader sample of patients compared to studies conducted so far, second only to the National Cancer Data base study in size, and with a similar distribution of patients between the two treatments. Of 656 patients with MIBC of various histologies, 325 were treated with $\mathrm{AC}$ and 331 with NC.

Our analysis shows a statistically significant difference in DFS in favor of NC (HR: 0.78, 95\% CI: 0.63-0.96, $P=0.02$ ), without any significant advantage in CSS (HR: 1.06, 95\% CI: 0.79-1.43, $P$ $=0.70)$ and OS (HR: $1.08 ; 95 \%$ CI 0.83-1.39, $P=0.57)$.

A possible explanation for the better DFS in the NC group may derive from the different pathological characteristics of the two groups. Indeed, in the adjuvant group, a significant percentage of 


\section{MIBC}

NC: median 34.6 months (25.3-43.9)

AC: median 24.9 months (19.4-30.5)

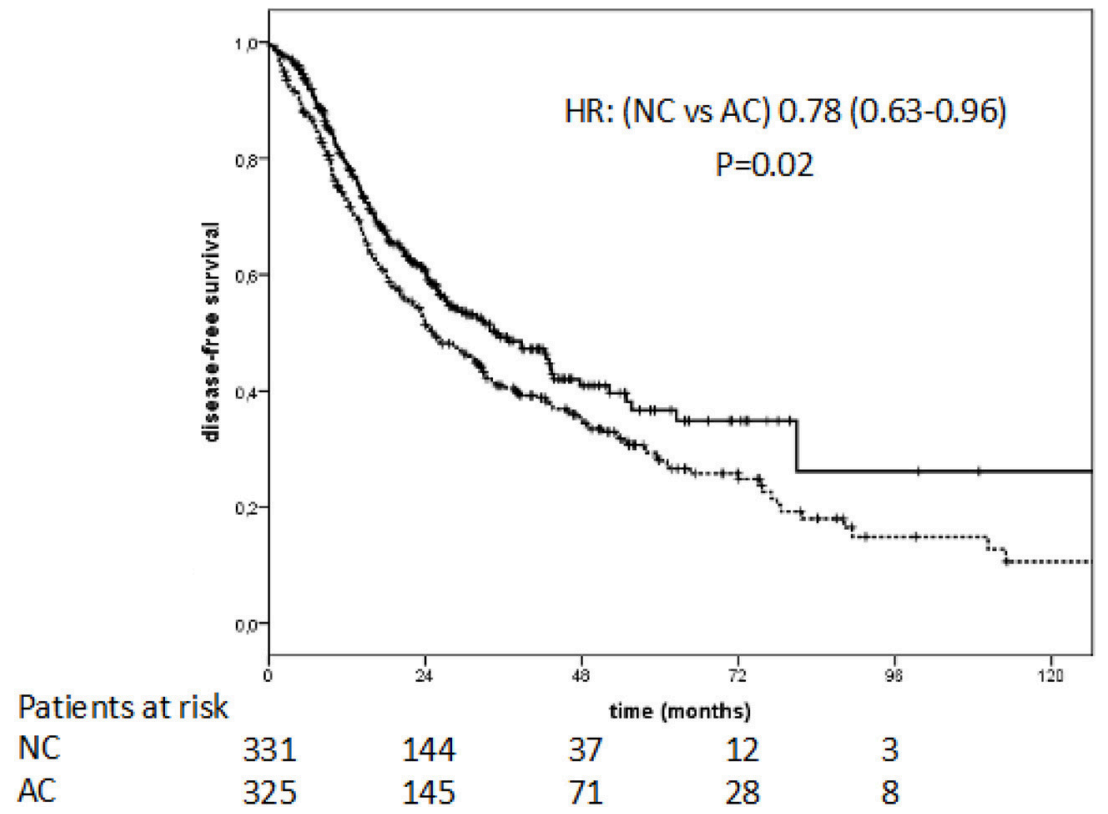

FIGURE 2 | Disease free survival in NC vs. AC treated patients with MIBC.

MIBC

NC: median 115.2 months (30.3-200.1)

AC: median 92.8 months (73.3-112.3)

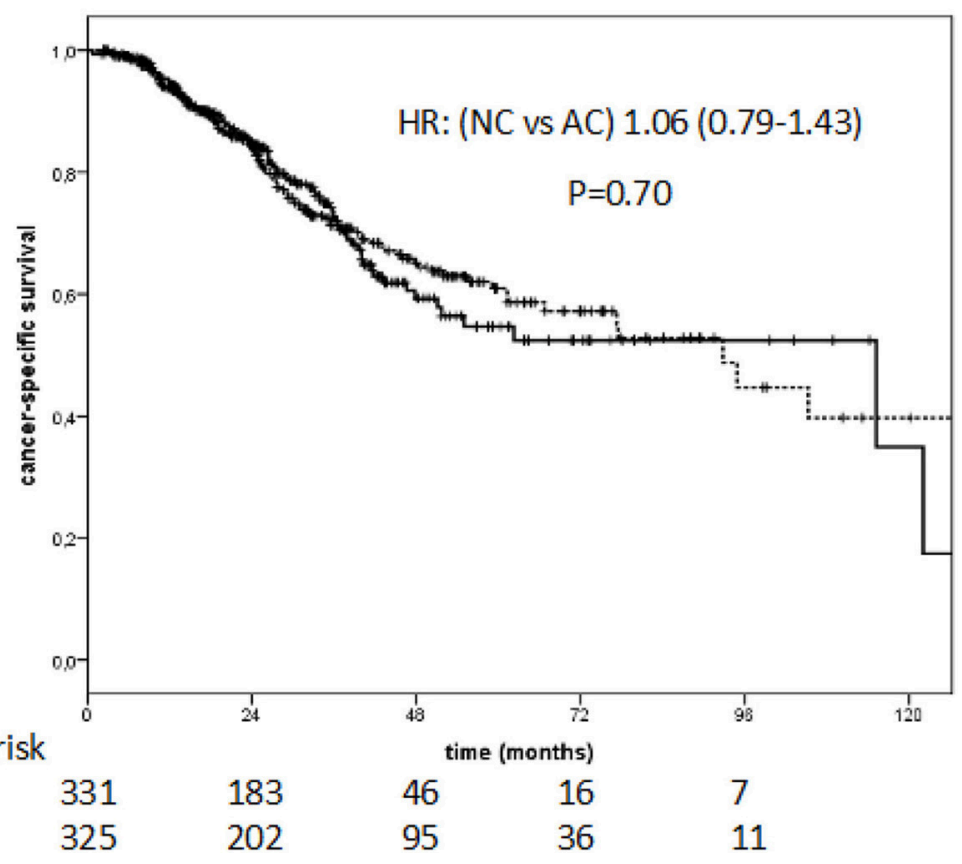

FIGURE 3 | Cancer specific survival in NC vs. AC patients with MIBC. 


\section{MIBC}

NC: median 51.7 months (37.8-65.6)

AC: median 66.8 months (51.1-82.5)

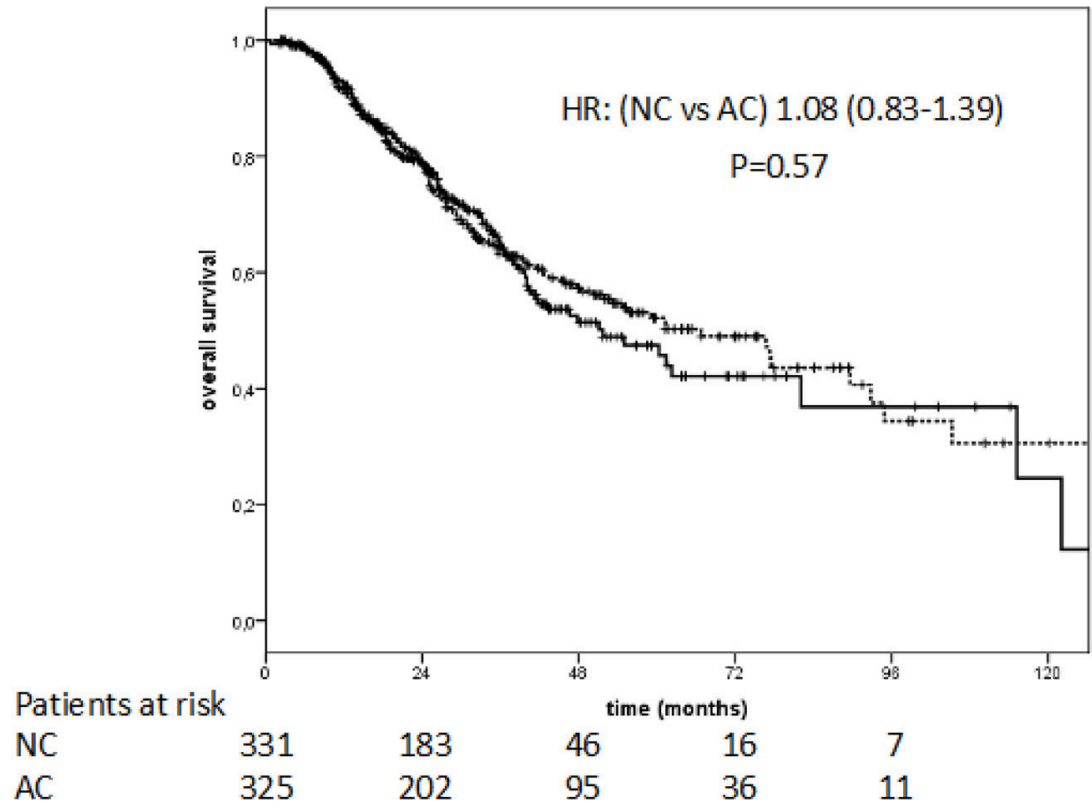

FIGURE 4 | Overall survival in NC vs. AC treated patients with MIBC.

patients had positive lymph nodes at diagnosis $(\mathrm{cN}+: 21.2 \%)$ and $21.2 \%$ of patients underwent surgery without data about lymph node status (cNx: 21.2\%). This could justify the worse DFS of the AC group, probably due both to inadequate staging and to greater disease burden, as these patients have metastatic disease at the time of surgery and diagnosis.

The advantage in DFS does not translate into a benefit in CSS and OS. The absence of a significant difference in OS and CSS may derive from the not-negligible percentage of patients with positive lymph nodes in the NC group ( $\mathrm{pN}+: 20.5 \%)$ and the number of patients without adequate pathological lymph node staging (pNx: $2.7 \%$ ). These patients may have a poorer outcome due to the lack of chemotherapy responsiveness, micrometastatic disease that would later progress or a greater disease burden. Indeed, many of these patients with $\mathrm{pN}+$ and $\mathrm{pNx}$ disease may have been under staged, and therefore not adequately treated.

Furthermore, the significance of DFS may be due to the higher number of events with respect to the number of deaths from disease considered in the CSS. When performing the Propensity Score matched for $\mathrm{pT}$ and $\mathrm{pN}$ all differences disappeared and $\mathrm{NC}$ and $\mathrm{AC}$ had a similar outcome.

Moreover, the presence of lymph node metastasis had a negative impact on outcomes in the NC group, with worse 5 year DFS and OS (DFS 5 years pN0 vs. pN+: 47 vs. $9 \%$, respectively, $P<0.0001$; OS 5 years pN0 vs. pN+: 51 vs. $25 \%$, respectively, $P$ $<0.0001)$. In the AC group, there are no significant differences in DFS $(P=0.11)$ or OS $(P=0.29)$ in relation to pathological lymph node status ( $\mathrm{pN} 0$ vs. $\mathrm{pN}+$ ). These results are not aligned with those of the EORTC adjuvant study, where the absence of lymph node involvement ( $\mathrm{p} \mathrm{N0}$ ) was associated with better OS compared to patients with positive lymph nodes $(\mathrm{pN}+)$.

Similar to past studies, we observed that pathological complete response to NC (ypT0) positively influenced both DFS and OS at 5 years compared to patients with residual disease $(\geq \mathrm{pT} 1$; $P:<0.0001)$. The percentage of complete pathological response ( $\mathrm{pCR}=\mathrm{ypT} 0)$ in the NC group was $27.5 \%$ slightly less than that in the pivotal SWOG study (pCR: 38\%), but aligned with other cisplatin-based NC prospective and retrospective studies which have ranged from $\sim 20$ to $38 \%$ (15-17).

Whether to give NC or AC to variant histologies is a clinically relevant question. In our study, variant histology and mixed forms (urothelial with a squamous component or other types) did not appear to affect 5-year DFS and 5-year OS in the NC ( $P$ : 0.15). However, in the AC group, patients with urothelial cancers did appear to have improved 5 -year OS (56 vs. $43 \%, P=0.001$ ) and 5-year DFS (31 vs. $19 \%, P=0.002)$ compared to patients with non-urothelial tumors.

In a secondary analysis of the SWOG S8710 study, the efficacy of NC was estimated in relation to histology, divided into urothelial tumors and mixed forms. There was a survival benefit for NC in patients with mixed histology (HR: 0.46, 95\% CI 0.25$0.87, P: 0.02$ ), as well as in those with urothelial tumors, although not statistically significant (HR: $0.90 ; 95 \%$ CI $0.67-1.21 ; P: 0.48$ ) (18). These results, together with those of our study, suggest that the presence of a non-urothelial component may not confer resistance to chemotherapy, and does not constitute an element of absolute exclusion from perioperative treatment. Better 5-year 
DFS and OS were /not was not obtained with urothelial tumors in the AC group. Given the retrospective nature of the analysis, this cannot lead to definitive conclusions.

Our study is one of the largest global analyses conducted so far comparing outcomes between NC and AC in MIBC across major international centers involved in the treatment of urogenital tumors and with experience in this field. Nonetheless, it has several limitations that derive mainly from the retrospective nature of the study, a potential bias in the distribution of patient characteristics and the type of statistical analysis, which does not allow definitive conclusions. A major limitation in this comparison is that $\mathrm{NC}$ is administered based on clinical staging whereas AC is given based on pathologic staging, making the comparison even more difficult. There is always the potential for heterogeneity in outcomes and understaging based on clinical staging.

Another problem is the lack of data concerning performance status in all patients, and the heterogeneity of chemotherapy treatments, often without cisplatin (more than $20 \%$ of patients treated without cisplatin in each group). Moreover, subsequent therapies for metastatic disease, that may have affected OS and CSS results, were not available for all patients.

Ongoing trials such as the VESPER trial (NCT01812369) of perioperative chemotherapy for patients with locally advanced bladder cancer, have the aim of comparing the efficacy of GC and HD-MVAC in terms of PFS in patients treated with perioperative treatment, both before and after surgery. Another trial conducted by the SWOG will use the CoeXpressionExtrapolatioN (COXEN) model to evaluate genetic

\section{REFERENCES}

1. Siegel RL, Miller KD, Jemal A. Cancer statistics, 2018. CA Cancer J Clin. (2018) 68:7-30. doi: 10.3322/caac.21442. Epub 2018 Jan 4.

2. Stein JP, LieskovskyG, CoteR, Groshen S, Feng AC, Boyd S, et al. Radical cystectomy in the treatment of invasive bladder cancer: long term resultsin 1054 patients. J ClinOncol. (2001) 19:666-75. doi: 10.1200/JCO.2001.19.3.666

3. Hautmann RE, Gschwend JE, de Petriconi RC, Kron M, Volkmer B. Cystectomy for transitional cell carcinoma of the bladder: results of a surgery only series in the neobladder era. J Urol. (2006) 176D2:486-92. discussion 491-2. doi: 10.1016/j.juro.2006.03.038

4. Kaplan E, Meier P. Nonparametric estimation from incomplete observations. J Am Stat Assoc. (1958) 53:457-81.

5. Griffiths G, Hall R, Sylvester R, Raghavan D, Parmar MK, Club Urologico Espanol de Tratamiento Oncologico Group, et al. International phase III trial assessing neoadjuvant cisplatin, methotrexate, and vinblastine chemotherapy for muscle-invasive bladder cancer: longterm results of the BA06 30894 trial. J ClinOncol. (2011) 29:2171-7. doi: 10.1200/JCO.2010.32.3139

6. Grossman HB, Natale RB, Tangen CM, Speights VO, Vogelzang NJ, Trump DL, et al. Neoadjuvant chemotherapy plus cystectomy compared with cystectomy alone for locally advanced bladder cancer. N Engl J Med. (2003) 349:859-66. doi: 10.1056/NEJMoa022148

7. Vale CL, Advanced Bladder Cancer (ABC) Meta-analysis Collaboration. Neoadjuvant chemotherapy in invasive bladder cancer: update of a systematic review and meta-analysis of individual patient data advanced bladder cancer (ABC) meta analysis collaboration. Eur Urol. (2005) 48:202-5, discussion 205-206. doi: 10.1016/j.eururo.2005.04.006

8. Paz-Ares L, Solsona E, Esteban E, Saez A, Gonzalez-Larriba J, Anton A, et al. On behalf of the SOGUG and GUO-AEU groups Randomized phase III trial comparing adjuvant paclitaxel/gemcitabine/cisplatin (PGC) profiling. Patients will be treated with either GC or HD M-VAC in the NCsetting (NCT02788201).

Moreover, the combination of multiple treatments, could be a way to achieve a better survival compared to surgery and perioperative chemotherapy. In a recent prospective randomized phase 2 study by Zaghloul et al. (19), AC with cisplatin and gemcitabine, was compared to the combination of AC plus radiotherapy, with a statistically significant improvement in locoregional recurrence free survival and a trend in better DFS and OS.

With new data arising from multiple TCGA analytical platforms, the hope in the future is that better molecular characterization of bladder cancers will help us to determine useful predictive factors to select patients who will benefit most from perioperative chemotherapy or other novel therapies (20).

\section{AUTHOR CONTRIBUTIONS}

GB and CS designed the analysis and wrote the manuscript. DG conducted the statistical analysis and contributed to the writing of the manuscript. FC, EP, LH, EY, SC, SP, AA, TP, UD, $\mathrm{NA}, \mathrm{AB}, \mathrm{SL}, \mathrm{AN}, \mathrm{UV}, \mathrm{GN}, \mathrm{JBe}, \mathrm{JBa}$, and MG have made direct and intellectual contributions to the work and approved it for publication.

\section{SUPPLEMENTARY MATERIAL}

The Supplementary Material for this article can be found online at: https://www.frontiersin.org/articles/10.3389/fonc. 2018.00463/full\#supplementary-material

to observation in patients with resected invasive bladder cancer: results of the SOGUG (Spanish Oncology Genito-Urinary Group) 99/01 study. J Clin Oncol. (2010) 28(Suppl. 18):LBA4518. doi: 10.1200/jco.2010.28.18_suppl.lb a4518

9. Sternberg CN, Skoneczna I, Kerst JM, Albers P, Fossa SD, Agerbaek M, et al. Immediate versus deferred chemotherapy after radical cystectomy in patients with pT3-pT4 or N1M0 urothelial carcinoma of the bladder (EORTC 30994): an intergroup, open-label, randomised phase 3 trial. Lancet Oncol. (2015) 16:76-86. doi: 10.1016/S1470-2045(14)71160-X

10. Leow JJ, Martin-Doyle W, Rajagopal PS, Patel CG, Anderson EM, Rothman AT, et al. Adjuvant chemotherapy for invasive bladder cancer: a 2013 updated systematic review and meta-analysis of randomized trials. Eur Urol. (2014) 66:42-54. doi: 10.1016/j.eururo.2013.08.033

11. Wosnitzer MS, Hruby GW, Murphy AM, Barlow LJ, Cordon-Cardo C, Mansukhani $\mathrm{M}$, et al. A comparison of the outcomes of neoadjuvant and adjuvant chemotherapy for clinical T2-T4aN0-N2M0 bladder cancer. Cancer (2012) 118:358-64. doi: 10.1002/cncr.26278

12. Millikan R, Dinney C, Swanson D, Sweeney P, Ro JY, Smith TL, et al. Integrated therapy for locally advanced bladder cancer: final report of a randomized trial of cystectomy plus adjuvant M-VAC versus cystectomy with both preoperative and postoperative M-VAC. JClin Oncol. (2001) 19:4005-13. doi: 10.1200/JCO.2001.19.20.4005

13. Matsubara N, Mukai H, Naito Y, Nezu M, Itoh K. Comparison between neoadjuvant and adjuvant gemcitabine plus cisplatin chemotherapy for muscle-invasive bladder cancer. Asia Pac J Clin Oncol. (2013) 9:310-7. doi: $10.1111 /$ ajco.12017

14. Sonpavde G, Nelson RA, Trinh QD, Agarwal N, Nix J, Kardos S, et al. Adjuvant versus neoadjuvant chemotherapy for muscle-invasive bladder cancer (MIBC): Analysis of the National Cancer Database (NCDB). J Clin Oncol.34(15_Suppl):4524. doi: 10.1200/JCO.2016.34.15_suppl.4524 
15. van de Putte EEF, Mertens LS, Meijer RP, van der Heijden MS, Bex A, van der Poel HG, et al. Neoadjuvant induction dose-dense MVAC for muscle invasive bladder cancer: efficacy and safety compared with classic MVAC and gemcitabine/cisplatin. World J Urol. (2016) 34:157-62. doi: 10.1007/s00345-015-1636-y

16. Choueiri TK, Jacobus S, Bellmunt J, Qu A, Appleman LJ, Tretter C, et al. Neoadjuvant dose-dense methotrexate, vinblastine, doxorubicin, and cisplatin with pegfilgrastim support in muscle-invasive urothelial cancer: pathologic, radiologic, and biomarker correlates. J Clin Oncol. (2014) 32:1889-94. doi: 10.1200/JCO.2013.52.4785

17. Plimack ER, Hoffman-Censits JH, Viterbo R, Trabulsi EJ, Ross EA, Greenberg RE, et al. Accelerated methotrexate, vinblastine, doxorubicin, and cisplatin is safe, effective and efficient neoadjuvant treatment for muscle-invasive bladder cancer: results of a multicenter phase II study with molecular correlates of response and toxicity. J Clin Oncol. (2014) 32:1895-901. doi: 10.1200/JCO.2013.53. 2465

18. Scosyrev E, Ely BW, Messing EM, Speights VO, Grossman HB, Wood DP, et al. Do mixed histological features affect survival benefit from neoadjuvant platinum-based combination chemotherapy in patients with locally advanced bladder cancer? A secondary analysis of Southwest Oncology Group-Directed Intergroup Study (S8710). BJU Int. (2011) 108:693-9. doi: 10.1111/j.1464-410X.2010. 09900.x

19. Zaghloul MS, Christodouleas JP, Smith A, Abdallah A, William H, Khaled HM, et al. Adjuvant sandwich chemotherapy plus radiotherapy vs. adjuvant chemotherapy alone for locally advanced bladder cancer after radical cystectomy. A randomized phase 2 trial. JAMA Surg. (2018) 153:e174591. doi: 10.1001/jamasurg.2017.4591

20. Robertson AG, Kim J, Al-Ahmadie H, Bellmunt J, Guo G, Cherniack $\mathrm{AD}$, et al. Comprehensive molecular characterization of muscle-invasive bladder cancer. Cell (2017) 171:540-556.e25. doi: 10.1016/j.cell.2017. 09.007

Conflict of Interest Statement: EP: Advisory Board: AstraZeneca, Bristol-Myers Squibb, Clovis, Exelixis, Genentech/Roche, Horizon Pharma, Incyte, Inovio, Janssen; Research Funding: Merck, Novartis, Pfizer, Eli Lilly and Co., Acceleron. LH: Advisory: Bayer, Genentech, Dendreon, Pfizer, Medivation/Astellas, Kew
Group, Theragene, Corvus, Merck, Exelixis; Research to the institution: Bayer, Sotio, Bristol-Myers Squib, Merck, Takeda, Dendreon/Valient, JannsenMedivation/Astellas, Genentech, Pfizer. EY: Consulting: Amgen, Bayer, Dendreon, Incyte, Janssen, Merck, QED, Seattle Genetics, Tolmar; Research funding to institution: Dendreon, Merck, Seattle Genetics. SC: Consulting/advisory: Roche, Clovis Oncology, Merck Research support: Astex Pharmaceuticals, Clovis Oncology. SP: Consulting: Genentech, Aveo, Eisai, Roche, Pfizer, Novartis, Exelixis, Ipsen, BMS, Astellas. TP: Honararia: Roche BMS AZ Merck; Research funding: AZ and Roche. UD: Advisory/Consultant: Bayer, Pfizer, Astellas, Janssen, Sanofi, BMS, Ipsen, Novartis. NA: Consultancy to Pfizer, Novartis, Merck, Genentech, Eisai, Exelixis, Clovis, EMD Serono, BMS, Astra Zeneca, Foundation One, Astellas, Ely Lilly, Bayer, Argos, Medivation, Clovis, Nektar; Research funding to my institution on my behalf: Active Biotech, Astra Zeneca, Bavarian Nordic, BMS, Calithera, Celldex, Eisai, Exelixis, Genetech, GSK (glaxosmithkline), Immunomedics, Janssen, Medivation, Merck, New link Genetics, Novartis, Pfizer, Prometheus, Rexahn, Sanofi, Takeda, Tracon. UV: Consulting: Bayer, Exelixis, BMS Inc.; Research support: Merck and Exelixis Inc.; GN: Research support4SC lectures: Pfizer, Pierre Fabre, Roche, MSD; advisory role: Roche, IMS Health, BMS, medac, MSD, Pfizer; travel grants: Roche Parma, Pfizer Pharma, BMS. JBe: Advisory: Bayer, Genentech, Pfizer, Medivation/Astellas, Merck, Asta-Zeneca, Exelixis; Research to the institution: Merck, Takeda, Pfizer. MG: Advisory Board: Merck, Genentech, BMS, Astra Zeneca, Pfizer; Research Funding: Novartis, Merck,Genentech, BMS, Astra Zeneca; CS: Research funding: Janssen, Lilly, Roche, Pfizer; Advisory Board: Lilly, BMS, Merck, Clovis, Pfizer.

The remaining authors declare that the research was conducted in the absence of any commercial or financial relationships that could be construed as a potential conflict of interest.

Copyright (c) 2018 Del Bene, Calabrò, Giannarelli, Plimack, Harshman, Yu, Crabb, Pal, Alva, Powles, De Giorgi, Agarwal, Bamias, Ladoire, Necchi, Vaishampayan, Niegisch, Bellmunt, Baniel, Galsky and Sternberg. This is an open-access article distributed under the terms of the Creative Commons Attribution License (CC BY). The use, distribution or reproduction in other forums is permitted, provided the original author(s) and the copyright owner(s) are credited and that the original publication in this journal is cited, in accordance with accepted academic practice. No use, distribution or reproduction is permitted which does not comply with these terms. 